Universidad de Lima

Facultad de Comunicación

Carrera de Comunicación

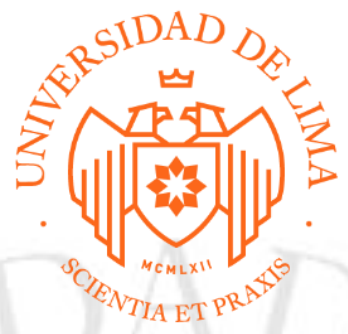

\title{
+ DECONSTRUCCIÓN
}

\section{- DISCRIMINACIÓN}

Trabajo de Suficiencia Profesional para optar el Título Profesional de

Licenciado en Comunicación

Anapaula Parodi Pestana

Código 20122061

Lucía Utano Díaz

Código 20121309

Lima - Perú

Mayo de 2019 


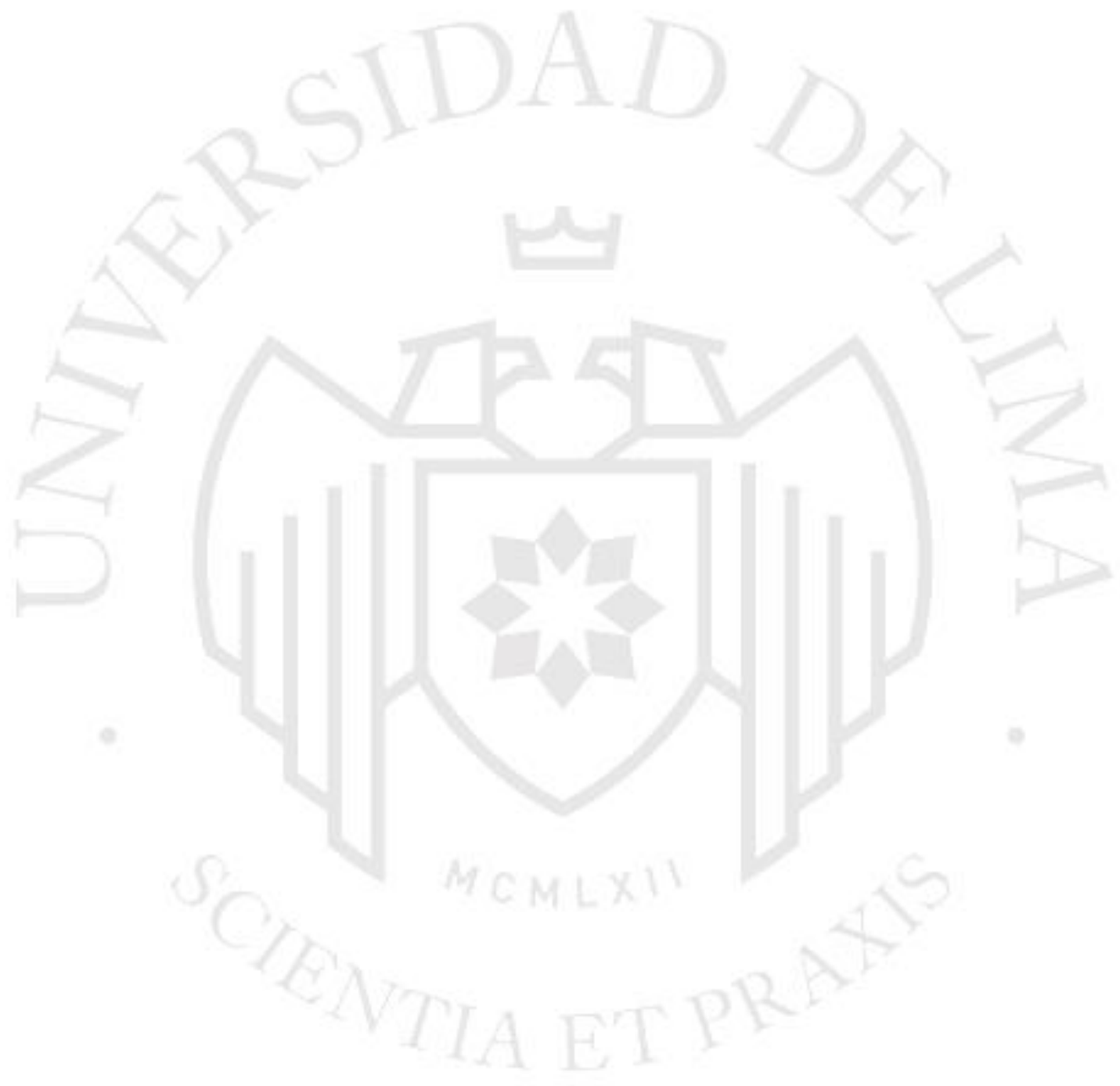


+ DECONSTRUCCIÓN

- DISCRIMINACIÓN 


\section{ÍNDICE}

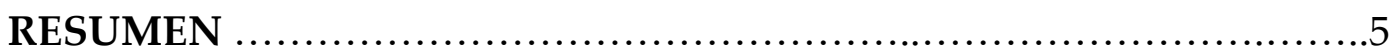

INTRODUCCIÓN y JUSTIFICACIÓN ................................6

1. ANTECEDENTES DEL TRABAJO ................................

1.1 Nociones básicas ...........................................

1.2 (De)Construcción social ....................................9

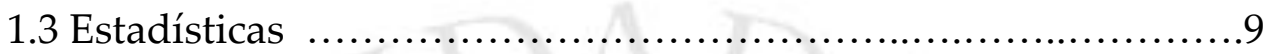

1.4 Discriminación : realidad y consecuencias .....................10

2. PROPUESTA DE COMUNICACIÓN Y SUSTENTACIÓN...............12

2.1 Ejes temáticos .............................................12

2.1.1 Qué es ser no binario.........................................12

2.1.2 Falta de educación y sociedad binaria hetero normativa .......12

2.1.3 Discriminación .............................................13

2.1.4 Conciencia ..............................................13

2.2 Personajes...............................................14

2.3 Propuesta audiovisual........................................15

3. RECOMENDACIONES ............................................16

4. PROPUESTA DE GUIÓN.............................................

4.1 Preguntas para personajes...................................17

4.2 Escaleta....................................................19

5. PLAN DE RODAJE............................................21

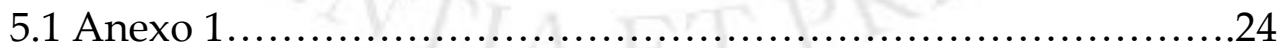

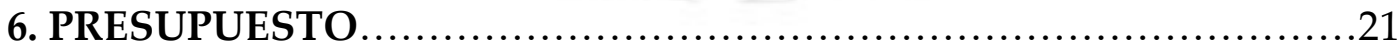

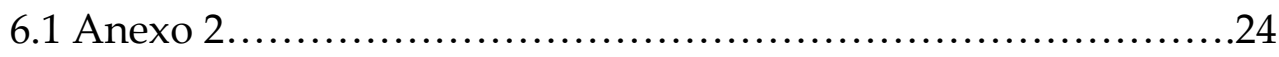

REFERENCIAS AUDIOVISUALES ...................................22

REFERENCIAS BIBLIOGRÁFICAS ................................... 22

MATERIAL DE ARCHIVO.........................................23

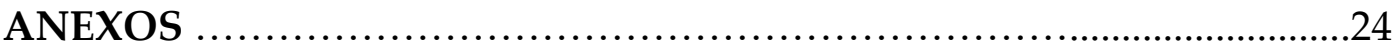




\section{RESUMEN}

Más deconstrucción menos discriminación, es un documental que analiza la discriminación a personas no binarias, que son parte de la comunidad LGBTQI. Por medio de entrevistas a expertos y a personas no binarias, se informa sobre lo que es ser no binario, se analiza la educación dentro del país y la sociedad binaria heteronormativa en la que vivimos, que desencadenan la discriminación. Así mismo, se trata de crear conciencia ante la ignorancia que existe actualmente frente a estas personas.

LINK: https:/drive.google.com/file/d/1Hz4Qqp0G619M1xW othj9IG0ggrPM$\underline{1 Z / v i e w ? \text { usp=sharing }}$ 


\section{INTRODUCCIÓN Y JUSTIFICACIÓN}

La discriminación en el Perú es un tema urgente que debería preocuparnos a todos. Al analizar las diferentes formas de discriminación, se encontró una cifra a bastante alarmante: el 62.7\% de la población LGBTQI peruana, asegura haber sufrido de discriminación, según el INEI (2017).

Dadas estas cifras, se decidió enfocarse en la comunidad LGBTQI. Una comunidad que mendiga derechos hasta el día de hoy y que no está siendo visibilizada en todas sus formas, muy a parte de que sea una minoría.

Dado que esta comunidad es bastante amplia, nos hemos centrado en las personas no binarias. Al hacer la investigación, descubrimos que existe mucha ignorancia con respecto a las personas no binarias, muchas dudas sobre la identidad y expresión de género y sobre la orientación sexual.

Pero, ¿por qué personas no binarias? Porque es necesario saber quiénes son, entenderles, aceptarles y educarnos para no cometer errores ni discriminar al relacionarnos o hablar de elles a causa de nuestra ignorancia.

La propuesta para desarrollar el documental fue hacerlo de forma lineal, simple, educatica e informativa, basada en conversaciones con personas no binarias como expertos en el tema. Se decidió hacerlo de esta forma porque de por sí es un tema complejo y no se quería opacarlo con que algo que por innovador resultara distractor. 


\section{ANTECEDENTES DEL TRABAJO}

\section{1 Nociones básicas}

Para inciar la investigación es importante familiarizarnos con términos que quizás no se tengan claros o se hayan escuchado. Se comenzará definiendo lo que significan las sigla LGBTQI:

- Lesbianas. mujeres que sienten atracción sexual, romántica y física por otras mujeres.

- Gay. Hombres que sienten atracción sexual, romántica y física por otros hombres. Esta palabra también se usa para refererise a las lebianas.

- Bisexual. Personas que se sienten atracción sexual, romántica y física tanto por hombres como mujeres.

- Trangénero. "Personas cuya identidad, apariencia y comportamiento se encuentra fuera de las normas de género convencionales." (Ibañez, 2017, p.10)

- Intersexual. Persona que tiene una anatomía sexual reproductiva que vaya con lo impuesto como femenino y masculino

- Queer. Personas que no se identifican con los términos tradicionales de sexualidad o género. Dentro de lo Queer se encuentran las personas no binarias, pero esto se definirá un poco más adelante.

Hay que dejar claro que esta sigla no es una regla y su contenido puede variar de acuerdo a las sociedad y contexto en la que se desenvuelva.

Para continuar hay que definir también lo que significa, sexo, género orientación sexual e identidad y expresión de género. Deberían ser términos que todos conozcan, pero lamentablemente no es así y aún crean mucha confusión. Para hablar de estos conceptos es necesario recurrir al los otros. Según Machuca, Cocchella y Gallegos (2016) cuando se habla de sexualidad y género, se hace uso de métodos con los cuales se categoriza la manera en que las personas viven, se 
expresan y relacionan. Si bien esa práctica es necesaria para construir conocimiento, puede también devenir en una limitación al momento de abordar las particularidades y diversidad de las personas (p. 24).

- Sexo: usado tradicionalmente para referir el tipo de genitalidad que determina el ser masculino o femenino de las personas al nacer.

- Género: De Martino (2009) dice que el género debe ser comprendido como una construcción cultural. Miller (2005) comenta que el género se entiende como el grado de femenidad o masculinidad que se encarnan en el cuerpo de acuerdo a las características de cada cultura.

- Orientacion sexual: se define en relación a los géneros hacia los se orienta la atracción afectiva, erótica, estética y/o romántica.

- Identidad de género: Según Ibañez (2017) es la sensación intima con la que las personas se identifican hacia un género, no necesariamente masculino o femenino, puede estar al medio o fuera de esas categorias.

- Expresión de género: formas en las que manifestamos nuestro género.

Por último, es necesario explicar lo que significa ser no binario. Machuca, Cocchella y Gallegos (2016) nos dicen que:

Las personas de género no binario son aquellas que no se identifican con el sexo diagnosticado al nacer y/o con el género asignado, sea de forma total o parcial. Es por esto que usualmente pensamos en ellxs como personas trans, aunque no necesariamente se identifiquen como hombres o mujeres trans, sino como personas cuya identidad y expresión de género no encaja ni se limita al binario. Esto puede deberse a que estas personas entienden su identidad de género como múltiple, fluida, indefinida, o porque esta representa una tercera opción de género que puede ser tanto femenina y masculina, o algo distinto (p. 104). 


\section{2 (De)Construcción social}

En la actualidad, nos encontramos en una sociedad binaria heteronormativa que hace que se rechace lo diferente, lo "anormal", nace de aquí la discriminación hacia cualquier tipo de persona que no cuadre dentro de los parámetros de lo socialmente instaurado.

Es fundamental hablar de deconstrucción, de la desconstrucción de lo establecido para poder avanzar como sociedad. Primero, para poder entenderla, hay que definir que es la construcción. Butler (2007), menciona que la autora Monique Witting ve en la naturaleza no una materialidad vigorosa, un medio, superficie u objeto, sino una «idea» creada y preservada con el objetivo del control social (p. 248). Esto implica un encasillamiento hacia las personas y un rechazo a lo que está fuera de ese control. Butler, líneas abajo, también menciona que Witting tiene como propósito explicar la idea de un cuerpo natural como una construcción y proponer una serie de estrategias deconstructivas/reconstructivas para configurar cuerpos que refuten el poder de la heterosexualidad (p. 248). En

el caso del género, este debe ser deconstruido desde su entendimiento como algo femenino o masculino, es decir, binario, mas no anulando estas indentidades sino aumentando los matices que hay entre ellos. Comprender que el género no es binario conllevaría haber logrado "la deconstrucción de constructos que siempre implican ya cierto tipo de violencia contra las opciones del cuerpo." (Butler, 2007, p. 250)

\section{3 Estadísticas}

El INEI realizó la primera encuesta a personas de la comunidad LGBTQI en el año 2017. Analizamos algunas cifras importantes: 
- Más de doce mil personas respodieron el estudio con edades que fluctuaban en su mayoría entre los 18 y 29 años.

- $\quad$ El 11.5 \% de esa población sufrió discriminación en el trabajo

- $\quad$ El $7.5 \%$ se considera del género no binario

- El $56.5 \%$ de esa población no expresa sin temor su identidad de género/orientación sexual y de aquí el $72.5 \%$ no lo hace por miedo a ser discriminado y/o agredido.

- Como se mencionó antes, el 62.7 \% de esta población ha sido discriminado en diferentes espacios como espacios públicos, ámbito educativo, medio de transporte, ámbito laboral, entre otros.

- De este último grupo, el 84.9 \% fue gritada, hostigada y/o amenzada y a un $26.2 \%$ los obligaron a cambiar de apariencia.

Estas cifras son alarmantes, pero necesarias de saber para conocer la situación por la que pasa el Perú. Las personas de género no binario se encuentran expuestas la mayor parte del tiempo a situaciones de discriminación.

\subsection{Discriminación: realidad y consecuencias}

Más del 60\% de la poblacion LGBTQI ha sido discriminada a lo largo de su vida, cifra preocupante, pero de la que no se habla. Sufrir un acto de discriminación puede ser muy traumático de acuerdo a la intensidad y complejidad de este. ¿Se encuentra la población LGBTQI segura para poder expresarse con libertad? No. ¿Y qué es lo que causan los actos de discriminación en la personas LGBTQI? Según la encuesta del INEI (2017), se sienten excluidas y aisladas, tienen sentimientos de culpa y/o impotencia, fatiga, intentos y/o pensamientos suicidas, falta de apetito, entre otras sensaciones y malestares. Desafortunadamente, solo una tercera parte de esta población acudió a un psicólogo y solo un $4.4 \%$ fue a denunciar el acto discriminatorio. Las cifras son tan bajas que hace reflexionar sobre la salud mental y el apoyo de las autoridades en el Perú. No existen muchos espacios de visibilización, afortunadamente existen organizaciones que se 
encargan de informar y acoger a personas que hayan sido discriminadas o violentadas para asesorarlas y brindarles ayuda. Estas instituciones son el Mhol, PROMSEX, No tengo miedo, etc. Esta última, tiene dos publicaciones: Estado de violencia y Nuestra voz persiste, gracias a las ganacias por estos libros y otras publicaciones más pequeñas, No tengo miedo ha podido alquilar una casa para funcionar adecuademente como la colectiva que son.

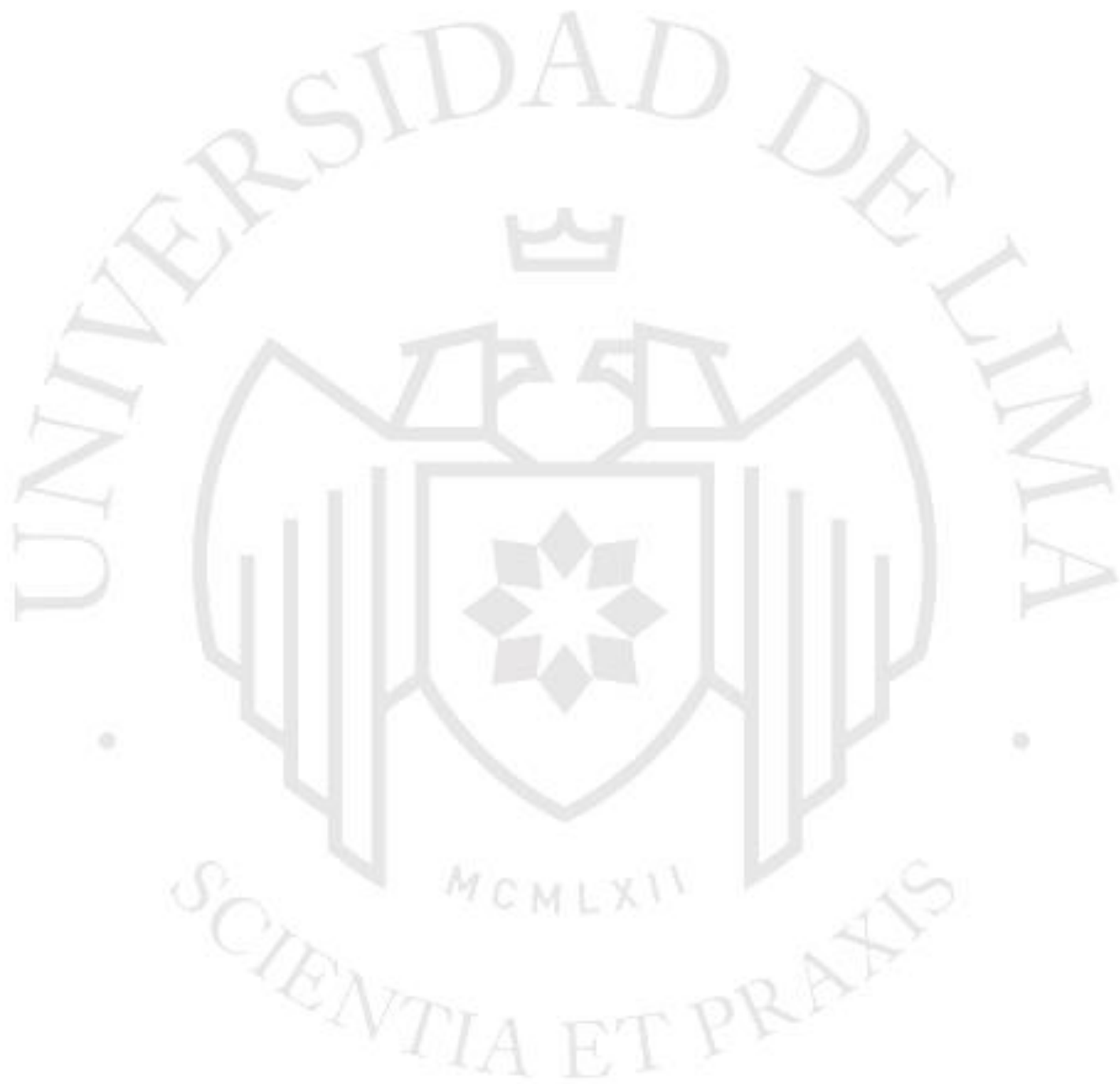




\section{PROPUESTA DE COMUNICACIÓN y SUSTENTACIÓN}

\section{1 Ejes temáticos}

Hemos dividido nuestra propuesta de comunicación en ejes temáticos relevantes al tema porque, al ser un documental corto, se quiere lograr informar y crear algún tipo de conciencia ante la falta de educación y la sociedad actual que desencadenan la discriminación.

\section{1. ¿Qué es ser no binario?}

Para poder poner en contexto al espectador desde un inicio, el documental nos explica, a través de una entrevista a un psicoterapeuta, lo que es un sistema de género binario, para luego ahondar en el no binarismo. Así mismo, se comienza con los testimonios de dos personas no binarias que relatan sus sentires y lo que los identifica con la no binariedad. Se decidió abarcar esta información en los primeros minutos del proyecto para poder, de cierta forma, entender estos términos, conocer a los personajes y comenzar a adentrarnos en las causas de la discriminación hacia los no binarios.

\section{1. 2 Falta de educación y sociedad binaria heternormativa}

Luego de explicar lo que significa ser una persona no binaria por medio de los entrevistados, se pasa a hablar de educación y sociedad. Se discrimina a lo diferente, a lo fuera de lo "normal" y lo normal es lo que nos impone la sociedad desde hace muchas décadas con la heteronormatividad.

La discriminación va estrictamente ligada a la educación y los entrevistados hacen incapié en lo pauperrima que lo es en el Perú. Es de suma importacia analizar el sistema en el vivimos. Uno de los entrevistados menciona que nos 
encontramos dentro de una discriminación sistémica, que funciona a nivel institucional, de espacio público, familiar, laboral, entre otras. Otro de los entrevistados dice que la sociedad actualmente no resiste el no binarismo y cambiar el proceso social, deconstruir los binarismos, es muy difícil para llegar a hacer la vida más amable.

Se decidió continuar por este camino narrativo para poder darle una base al espectador sobre las causas de la discriminación a las personas no binarias.

\section{1. 3 Discriminación}

El tema de discriminación se toca a lo largo de todo el documental. Se decidió no centrarse en un caso específico como para poder englobar todo lo que implica la discriminación hacia la comunidad LGBTQI y en particular hacia las personas no binarias. Se llegó a este planteamiento ya que, al ser un documental que tiene como principales objetivos educar y concientizar, es mejor tener una mirada más amplia de la problemática.

\section{1. 4 Conciencia}

En los últimos minutos del documental, lo que se desea es concientizar a la audiencia ante el problema. Con los testimonios de expertos y personas no binarias se desea hacer visible que la discriminación está presente siempre, desde preguntas tan simples como ¿qué eres?, ¿por qué eres así?, etc, hasta los insultos, la violencia y abuso de poder. Criticando la educación que el Perú tiene en estos momentos, que no se encuentra ni cerca de poder incluir lo que propone el enfoque de género dentro de la currícula nacional.

A través de este documental se quiere dejar claro que existen minorias sexuales, como los no binarios, que tienen que ser reconocidas y respetadas por igual en todos sus ámbitos, pero aún queda mucho por hacer. 
También dejar ver que esta minoria se ve afectada psicológica y emocionalmente ante la discriminación, como Mili Palacios, que tuvo que cerrar todas sus redes sociales por el bullying cibernético que causaba que sea una persona no binaria, que lamentablemente, las personas no entienden qué es serlo y al ignorarlo, se margina y ataca.

\section{2 Personajes}

Se cuenta con cuatro testimonios: dos expertos y dos personas no binarias.

- Diego García es un psicoterapeuta graduado de la Universidad de Lima que nos explica sobre la binariedad y la no binariedad. También nos habla de algunas causas por las que se da la discriminación desde su punto de vista psicológico.

- Patricia Ruiz Bravo es la Decana de la facultad de ciencias sociales de la Pontificia Universidad Católica del Perú. Ella nos da una mirada más amplia de la educación y nos cuenta que estamos muy lejos de llegar a la igualdad y al respeto ante lo diferente.

- Rudi Cocchella es antropóloga de la PUCP y cordinadora de la colectiva No tengo miedo. Ella es una persona no binaria que cuenta sus sentires y su persepectiva de la sociedad actual.

- Daniel Ramirez es comunicador egresado de la Universidad de Lima y no binario. Él narra sus experiencias en la escuela y nos da su punto de vista ante este problema.

Tanto Rudi como Daniel describen cómo fue que se identificaron como personas no binarias, para así poder hacer este término más entendible al espectador. 


\section{3 Propuesta audiovisual}

Se decidió hacer el documental con planos fijos por ser un documental testimonial. Se ha trabajado con tonos cálidos para dar una sensación más íntima y hogareña y así complemetar nuestro método de entrevista que más allá de ser de pregunta y respuesta fue más una conversación amical.

La música utilizada es calmada y melodiosa, es solo instrumental para que no distraiga de lo que está diciendo el entrevistado. Acompaña los relatos dándoles armonía y ligereza.

Las tomas de apoyo incluidas ayudan a contextualizar y hacer más dinámico el documental, también mostrar a los no binarios en una situación diferente a lo de la entrevista.

Siendo el tiempo del documental tan limitado se ha hecho una edición segmentada por ejes temáticos que permita entender todo lo expuesto. Se enfocó poner solo las respuestas que ayuden al documental. Se ha querido lograr un hilo de entendimiento que lleven al espectador a comprender todo lo que abarca la discriminación a las personas no binaria. 


\section{RECOMENDACIONES}

Para este proyecto se cree necesaria su extensión. Se quieren hacer dos versiones del proyecto una corta, que es la presentada y una más amplia que abarque el tema más a profundidad, ya que existe mucho material para aumentar la información tocada.

Se desea también mostrarlo en festivales para que alcance públicos nacionales y extranjeros. Y luego de esto, realizar una difusión masiva.

Se quiere buscar una alianza con No tengo miedo para que también puedan darle visibilidad dentro de sus plataformas y actividades. 


\section{PROPUESTA DE GUIÓN}

\section{1 Preguntas para personajes}

Preguntas para la sociológa:

1. ¿Sabes qué es una persona no-binaria?

2. Está dentro de la curricula escolar enseñar/visibilizar los diferentes tipos de personas que se encuentran dentro de la comunidad LGTBIQ a la cual pertenecen las personas no-binarias

3. Crees que la falta de educación sobre la comunidad LGTBIQ incite a la discriminacion

4. Cuáles son los índices de discriminación en los colegios a los niños que forman parte de la comunidad LGTBIQ

5. En el Libro "Elijo ser Yo" la autora Lucy Ibañez menciona "Creo que muchas veces rechazamos lo que somos o a los demás porque hemos aprendido a rechazar lo que es diferente a lo que estamos acostumbrados". Crees que el no visibilizar a temprana edad las diferentes orientaciones sexuales, expresiones e indentidades de género; puede llevar al futuro adulto a tener más rechazo a lo "diferente" a tal punto de discriminarlo.

\section{Preguntas para el psicoterapeuta:}

1. Nos puedes definir los términos orientación sexual, identidad de género y expresión de género

2. ¿Qué significa ser una persona de la comunidad LGTBQI?

3. Dentro de la comunidad LGTBQI, en dónde se encuentran las personas no binarias? (Explicar teoria queer y año de aprobacion/utilización del termino) 
4. ¿Qué significa ser/sentirte no binario?

5. ¿Cómo alguien llega a identificarse como no binario?

6. ¿Por qué existe rechazo/discriminacion hacia personas no binarias?

7. ¿Cómo afecta psicológicamente y emocionalmente ser no binario y ser discriminado por serlo?

\section{$\underline{\text { Preguntas para las personas no binarias }}$}

1. ¿Qué te identifica como una persona no binaria?

2. ¿Cómo te enteraste del término no binario?

3. ¿Desde cuando te identificas como una persona no binaria?

4. ¿Cómo fue para ti explicarle a tu familia el ser no binario?

5. ¿Cómo te ha afectado ser no binario?

6. ¿Has sido discriminado por ser no binario? ¿Qué pasó?

7. ¿De dónde crees que se origina la discriminación hacia las personas LGBTQI?

8. ¿Crees que nos educan aceptando la diversidad sexo/ género?

9. ¿Cómo prefieres que se refieran a ti?

10. ¿Te sientes visible en la sociedad en la que vivimos?

11. ¿Conoces a más personas no binarias? (hablar sobre No Tengo Miedo 


\subsection{Escaleta}

INTRO - LUGAR Y AÑO

INT. Casa Daniel. NOCHE.

Presentación del 1er personaje no binario.

INT. Casa Diego. DÍA

Explicación de lo binario y no binario por parte de un psicólogo.

INT. Casa de Chu. Noche

Presentación del 2do personaje no binario.

INT. Casa Diego. DÍA

Explicación de la comunidad LGBTQI.

INT. Casa de Chu. NOCHE

Rudi explica que la identidad de género es un proceso y da pie al tema de la educación.

INT. Casa Daniel. NOCHE

Daniel habla de su experiencia en el colegio como persona no binaria.

INT. Casa Patricia. NOCHE

Patricia habla sobre la falta de educación respecto a la diversidad sexo-género.

INT. Casa Diego. DÍA 
Explicación de cómo se genera la discriminación.

INT. Casa de Chu. NOCHE

Rudi menciona que la discriminación sucede de manera sistémica.

INT. Casa Daniel. NOCHE

Daniel habla sobre la ignoracia y cómo esta genera rechazo hacia lo diferente INT. Casa Patricia. NOCHE

Patricia comenta que vivimos en una sociedad que no resite el no binarismo y que la única forma de lograr que esto suceda es a través de la deconstucción.

INT. Casa Daniel. NOCHE

Daniel menciona cómo limitar la expresión de la personas limita también otras formas de expresión como el arte.

INT. Casa de Chu. NOCHE

Rudi habla de las diferentes maneras de expresar la identidad de género.

Comenta el caso de Mili Palacios, persona no binaria agredida en redes sociales. Termina mencionando lo obsena que es la obsesión de las personas hacia los cuerpos.

INT. Casa Daniel. NOCHE.

Daniel comenta sobre la falta de espacios físicos para llegar a una visibilidad de las personas que escapan de la heteronormatividad. Menciona que la colectiva No tengo miedo da ese espacio a las personas.

OUTRO - TÍTULO DEL DOCUMENTAL 


\section{PLAN DE RODAJE}

Anexo 1

6. PRESUPUESTO

Anexo 2 


\section{REFERENCIAS AUDIOVISUALES}

- MUCH PRIDE I Ep 4. Non-Binary: diálogo con una persona no binaria que explica lo que implica serlo. Nos basamos en esta entrevista por la metodología de utilizada, que es más una conversación, que fue lo que se realizó pero no mostramos la voz ni al entrevistador.

- Being Non-Binary: Documental de una persona no binaria en un momento específico de su vida. Sirvió como inspiración para poder filmar a los entrevistados en espacios que son parte de su intimidad

- 9trans: es un film documental sobre la experiencia de 9 personas transexuales, no binarias y de género neutro de Barcelona. Es una de la fuentes principales para crear el estilo del documental.

\section{REFERENCIAS BIBLIOGRÁFICAS}

Butler, J. (2007). El género en Disputa. Edición en castellano, pp. 248-250.

Butler, J. (2006). Deshacer el género. Edición en Castellano.

De Martino, M. (2009). Infancia Familia y Género. Primera edición.

Ibañez, L. (2017). Elijo ser yo. Primera edición, pp. 10.

Machuca, M., Cocchella, R., Gallegos, A. (2016). Nuestra voz persiste. Primera edición, pp. 24, 104.

Miller, J. (2005) Language potentials and gender ambiguity: Transgenderism and the role of social work. Gay E Lesbian Issues and Psychology Review. 1(1). http://www.researchgate.net/publication/26436448 language potentials and g ender ambiguity transgenderism and the role of social work 
INEI. (2017). Primera encuesta virtual para personas LGBTI.

\section{MATERIAL DE ARCHIVO}

- $\quad$ https://www.youtube.com/watch?v=V1RwSTCIzVg\&t=22s

- https://www.youtube.com/watch?v=EKgfHc6umRU\&t=53s

- $\quad$ https://www.youtube.com/watch?v=Q-myqQvihdE\&t=3s

- https://www.youtube.com/watch?v=7pvLDHFCEWk\&t=54s

- https://www.youtube.com/watch?v=kVe8wpmH IU

- https://www.youtube.com/watch?v=ruST0GPi68s\&t=2s

- $\quad$ https://www.facebook.com/marchadelorgullolima/videos/1846662658755 $\underline{880 /}$ 


\section{ANEXOS}

\section{1 Anexo 1}

\begin{tabular}{|c|c|c|c|}
\hline \multicolumn{4}{|c|}{ PLAN DE RODAJE } \\
\hline \multicolumn{4}{|c|}{ DIA 1: 27 DE ABRIL } \\
\hline \multicolumn{4}{|c|}{ LOCACIÓN 1: SURCO } \\
\hline HORA & PERSONAJE & DESCRIPCIÓN & NECESIDADES \\
\hline 10:00-10:30 AM & \multicolumn{3}{|c|}{ TRANSLADO ENTREVISTADO } \\
\hline $10: 30-12: 00 \mathrm{PM}$ & DIEGO & ENTREVISTA & Cámara, pechero, rhode \\
\hline \multicolumn{4}{|c|}{ DIA 2: 28 DE ABRIL } \\
\hline \multicolumn{4}{|c|}{ LOCACION 2: MIRAFLORES } \\
\hline 7:00 -7:30 PM & \multicolumn{3}{|c|}{ TRASLADO A LOCACIÓN } \\
\hline 7:30 - 8:00 PM & & SETEO & Cámara, pechero, rhode \\
\hline $8: 00-8: 40$ PM & PATRICIA & ENTREVISTA & cámara, pechero, rhode \\
\hline \multicolumn{4}{|c|}{ DIA 3: 29 DE ABRIL } \\
\hline \multicolumn{4}{|c|}{ LOCACIÓN 3: CASA CHU MAGDALENA } \\
\hline 7:00 - 7:45 PM & \multicolumn{3}{|c|}{ TRASLADO A LOCACIÓN } \\
\hline $7: 45-8: 30 \mathrm{PM}$ & & SETEO & Cámara, pechero, rhode \\
\hline $8: 30-9: 15$ PM & RUDI & ENTREVISTA & Cámara, pechero, rhode \\
\hline \multicolumn{4}{|c|}{ DIA 4: 1 DE MAYO } \\
\hline \multicolumn{4}{|c|}{ LOCACIÓN 4: MAGDALENA } \\
\hline $5: 30-6: 00 \mathrm{PM}$ & \multicolumn{3}{|c|}{ TRASLADO A LOCACIÓN } \\
\hline $6: 00-6: 30 \mathrm{PM}$ & & SETEO & Cámara, pechero, rhode \\
\hline $6: 30$ - 7:30 PM & DANIEL & ENTREVISTA & Cámara, pechero, rhode \\
\hline
\end{tabular}

\section{1 Anexo 2}

\section{PRESUPUESTO DOCUMENTAL}

\section{SÁBADO 27, DOMINGO 28, LUNES 29 DE ABRIL Y MIÉRCOLES 1 DE MAYO}

\begin{tabular}{|l|r|c|}
\hline & MONTO S/. & DESCRIPCIÓN \\
\hline MOVILIDAD & 60 & Traslados entrevistados/equipos \\
\hline COMIDA & 35 & Regalo para entrevistados \\
\hline EQUIPOS & 840 & Alquiler de cámara, luces, zoom y Rhode \\
\hline EXTRAS & 50 & compra de fluorescente \\
\hline TOTAL & \multicolumn{3}{|c|}{985} \\
\hline
\end{tabular}

